\title{
Sub-percent precision Møller polarimetry in experimental Hall C
}

Joshua Magee ${ }^{* \dagger}$

College of William and Mary

E-mail: jmagee@jlab.org

Modern experiments in Jefferson Lab Hall C require precise knowledge of the electron beam polarization. This knowledge comes from two complementary polarimeters (Møller and Compton). The Hall C Møller polarimeter uses a pure iron foil that is brute-force polarized out-of-plane by a superconducting solenoid. A system of quadrupole magnets focuses the scattered and recoil electrons onto a pair of calorimeters. This design offers several improvements compared to previous devices, enabling sub-percent systematic uncertainties. This talk will introduce the Hall $\mathrm{C}$ Møller polarimeter and discuss the necessary work to determine $<1 \%$ precision, using the $Q_{\text {weak }}$ experiment as an example.

XVth International Workshop on Polarized Sources, Targets, and Polarimetry September 9 - 13, 2013

Charlottesville, Virginia, USA

\footnotetext{
* Speaker.

${ }^{\dagger}$ Advisers: David Gaskell, Jefferson Lab and David Armstrong, College of William and Mary.
} 


\section{Introduction to the Hall C Møller}

The Basel Møller polarimeter in Jefferson Lab Hall C improved upon previous polarimeter designs substantially. The apparatus consists of the following components:

- A pure iron-foil target. The magnetic properties of pure iron are well understood

- A superconducting solenoid magnet which is used to brute-force polarize the pure iron target. The target polarization is out-of-plane. The solenoid is run at $3.5 \mathrm{Tesla}$; pure iron magnetically saturates at 2.2 Tesla.

- Two quadrupoles acting as an optical lens. These focus the $90^{\circ}$ center-of-mass Møller electrons, where the asymmetry is largest, onto the detector array. Both scattered and recoil electrons are required.

- Two calorimeters detect the scattered and recoil electrons in coincidence. A narrow timing gate of $5 \mathrm{~ns}$ reduces backgrounds from Mott scattering.

- A set of moveable collimators between the two quadrupoles. These are used to further reduce background and do not affect the acceptance.

Figure 1 shows the original layout and the layout for the recently completed $Q_{\text {weak }}$ experiment. Before $Q_{\text {weak }}$, an additional quadrupole was added to the beamline in preparation for the upcoming Jefferson Lab $12 \mathrm{GeV}$ upgrade. It was un-powered during the $Q_{\text {weak }}$ experiment.

For more information on specific hardware and design of the Hall C Møller polarimeter, the interested reader is directed elsewhere to $[1,2]$.
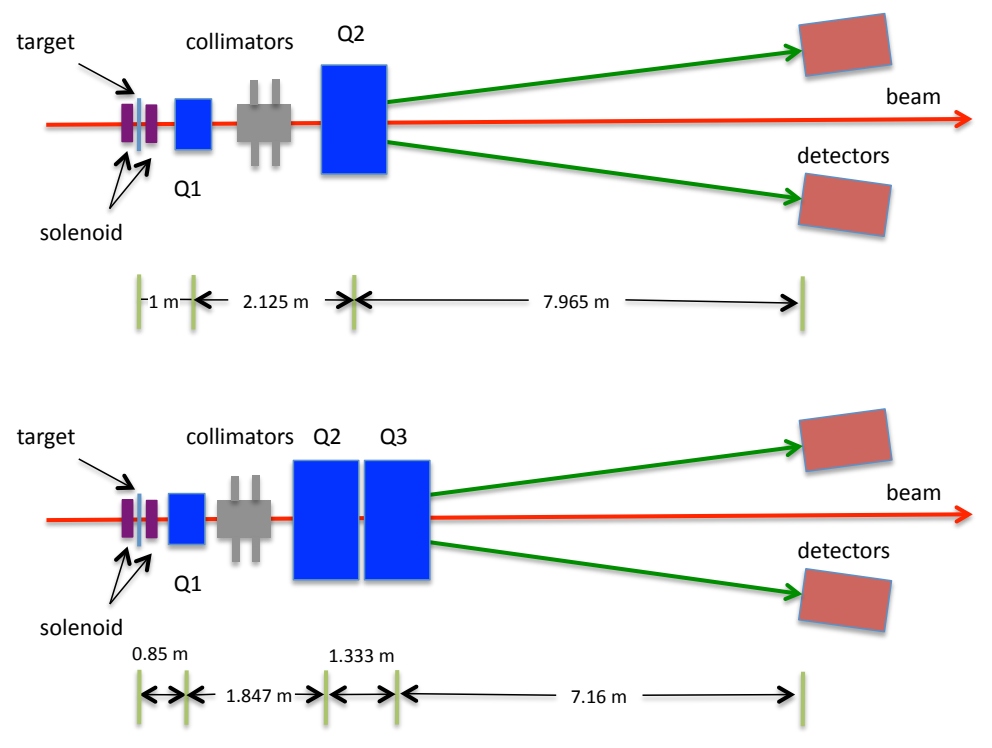

Figure 1: Layout of the Møller polarimeter in its original configuration (top) and after modification just prior to $Q_{\text {weak }}$ (bottom). During $Q_{\text {weak }}$ Q2 was unpowered; only Q1 and Q3 were used. 


\section{Møller Polarimeter systematic uncertainties}

As an example of the Hall C Møller polarimeter performance, the overall systematic uncertainty achieved for the recently completed $Q_{\text {weak }}$ experiment was $0.84 \%$. The systematic uncertainties are further broken down into two subcategories: those of "scale" and those called point-topoint. The scale uncertainties are systematics common to the whole run period. For instance the position of the immoveable collimators remains constant, as do the uncertainties due to radiative effects. Point-to-point uncertainties are those which vary between measurements, such as the beam position on target. Some systematics are split between the two when appropriate. See Table 1 for specific details.

From Table 1, note the following:

- The largest systematic uncertainty is from beam position on target. Several position sensitivity studies were performed during $Q_{\text {weak }}$, and are discussed below in Section 4.1.

- The uncertainties due to the quadrupole currents, and therefore magnetic fields, are particularly interesting and important, albeit small. They are discussed in detail later in Section 4.2.

- The Levchuk effect (scattering from the inner, unpolarized electron shells) is also sizeable. We take the uncertainty to be $10 \%$ of the overall effect.

- Lastly, a generous uncertainty is given to a "high-current extrapolation." Møller measurements are typically taken at low currents $\leq 2 \mu \mathrm{A}$, while $Q_{\text {weak }}$ production running was at $180 \mu \mathrm{A}$. Previous studies using a Møller beam raster, a kicker system[3, 4], and a special "beat frequency" study [5] have all shown no current dependence of the polarization up to 60 $\mu A$. Previously, a correlation was seen between photo-cathode quantum efficiency (QE) and polarization. It is possible that higher laser powers used in production running could result in a different effective $\mathrm{QE}$ than when performing low-current Møller measurements. However, during $Q_{\text {weak }}$ the $\mathrm{QE}$ was deliberately kept high. No other mechanism has been suggested to lead to any current dependence of polarization. To be conservative a large $0.5 \%$ uncertainty is given nonetheless.

\section{Møller Monte Carlo Simulation}

To determine the analyzing power and the effect of certain radiative corrections, a Monte Carlo simulation is used. It is a simple aperture checking device, rather than a complex GEANT-type simulation. The Møller Monte Carlo was originally based on the work in [6], and then customized for Jefferson Lab. Recently it has undergone substantial improvements, primarily focused on the magneto-optical elements, including: separating the entrance and exit half-solenoid TRANSPORT matrices for the incoming and scattered electrons[7, 8], and implementing 2nd-order TRANSPORT matrices to improve magneto-optical transport through the quadrupoles[8]. 


\begin{tabular}{|l|c|c|c|c|}
\hline Source & Uncertainty & $\mathrm{dA} / \mathrm{A}(\%)$ & Scale & Point-to-point \\
\hline Beam position X & $0.2 \mathrm{~mm}$ & 0.14 & - & 0.14 \\
\hline Beam position Y & $0.2 \mathrm{~mm}$ & 0.28 & - & 0.28 \\
\hline Beam angle X & $0.5 \mathrm{mrad}$ & 0.10 & - & 0.10 \\
\hline Beam angle Y & $0.5 \mathrm{mrad}$ & 0.10 & - & 0.10 \\
\hline Q1 current & $2 \%$ & 0.07 & - & 0.07 \\
\hline Q3 current & $1 \%$ & 0.05 & - & 0.05 \\
\hline Q3 position & $1 \mathrm{~mm}$ & 0.10 & 0.01 & - \\
\hline Multiple scattering & $10 \%$ & 0.01 & 0.01 & - \\
\hline Levchuk effect & $10 \%$ & 0.33 & 0.33 & - \\
\hline Fixed collimator positions & $0.5 \mathrm{~mm}$ & 0.03 & 0.03 & - \\
\hline Target temperature & $100 \%$ & 0.14 & 0.14 & - \\
\hline B-field direction & $2 \mathrm{degrees}$ & 0.14 & 0.14 & - \\
\hline B-field strength & $5 \%$ & 0.03 & 0.03 & - \\
\hline Spin polarization in Fe & - & 0.25 & 0.25 & - \\
\hline Electronic D.T. & $100 \%$ & 0.045 & 0.045 & - \\
\hline Solenoid focusing & $100 \%$ & 0.21 & 0.21 & - \\
\hline Solenoid position (x,y) & $0.5 \mathrm{~mm}$ & 0.23 & $0.23 / \sqrt{2}$ & $0.23 / \sqrt{2}$ \\
\hline High-current extrapolation & - & 0.50 & $0.50 / \sqrt{2}$ & $0.50 / \sqrt{2}$ \\
\hline Monte Carlo statistics & - & 0.14 & - & - \\
\hline Total & & 0.84 & 0.64 & 0.53 \\
\hline
\end{tabular}

Table 1: Final Møller systematic uncertainty list for the $Q_{\text {weak }}$ experiment. Note the dominant experimental systematic is beam position on target, and the conservative inclusion of high-current extrapolation uncertainty. The last two columns separate the systematic uncertainty in two parts: "scale" denotes systematics common to all data, while "point-to-point" are time-dependent contributions which apply to each measurement.

The simulation produces the polarimeter's analyzing power, which is used to extract the measured beam polarization. To validate the simulation, the predicted position sensitivities are compared to real-world beam position scans. Disagreements indicate improper implementation. Figure 2 in Section 4.1 has more detail on this process.

\section{Examples of systematic uncertainty studies}

The Møller device routinely took $0.5 \%$ statistical measurements in about 5 minutes. With 3 runs per measurement, the full statistical contribution for each measurement was about $0.3 \%$. In this section a few examples of the systematic uncertainties listed in Table 1 are discussed in some detail. 


\subsection{Position and angle systematic uncertainty}

Position and angle sensitivity of the beam on target is the Møller polarimeter's largest systematic uncertainty for $Q_{\text {weak }}{ }^{1}$. Because of the low beam energy required by $Q_{\text {weak }}$, the analyzing power can change $0.5 \% / \mathrm{mm}$.

Figure 2 shows the relative rates taken from a position sensitivity scan. Note that positions up to $\pm 2.0 \mathrm{~mm}$ were included; however, Møller production running was only performed within \pm 1 $\mathrm{mm}$ in both $x / y$. Data and simulation agree within this region.

The position uncertainty listed in Table 1 was determined from the actual position correction applied. The position correction depended on three particular things:

- The dependence of the measured polarization on beam position

- The uncertainty of projecting from the beam position monitors $(B P M \mathrm{~s})$ to the target

- The instrumental uncertainty in absolute BPM position

The dependence of the measured polarization on beam position was determined from simulation. Once the data/simulation relative rates agree, the simulation was used to determine the actual position sensitivity $(\partial P / \partial X)$ in both $X, Y$ position and angle on target. The simulated position sensitivity in $X$ is seen in Figure 3. It is interesting to note that the analyzing power seems to have such interesting non-linear behavior at the tails. This was also seen for the $Y$ sensitivity.

To determine the uncertainty involved in transporting from BPMs to the target, we compared various transport algorithms. Ultimately, comparing a straight projection, assuming no optical elements along the beamline, to a multi-step transport algorithm based on beamline geometry and entrance solenoid field was used to determine the uncertainty in electron transport to target. To determine sensitivity from the absolute $B P M$ position, we estimated knowledge of position to 0.1
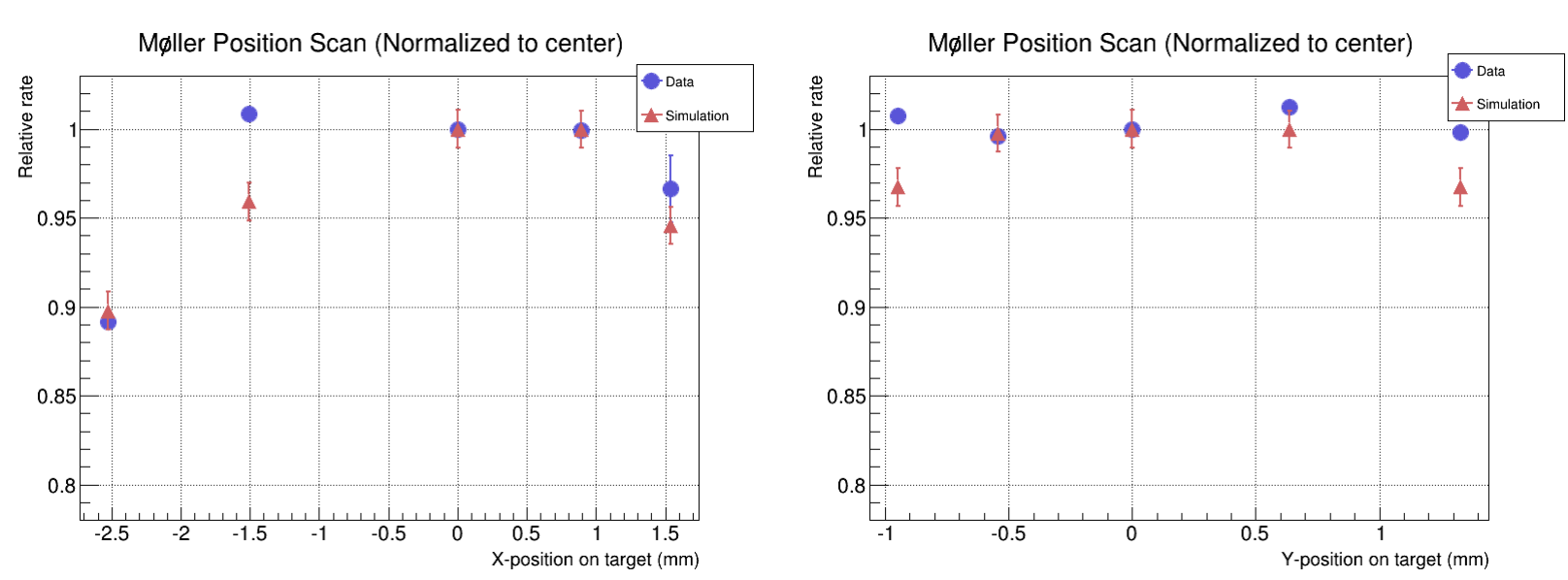

Figure 2: Comparison of the actual and simulated position scan. Note the relative agreement through $\pm 1 \mathrm{~mm}$. All production Møller measurements were within this range.

\footnotetext{
${ }^{1}$ Position and angle sensitivity are typically smaller at higher energies.
} 

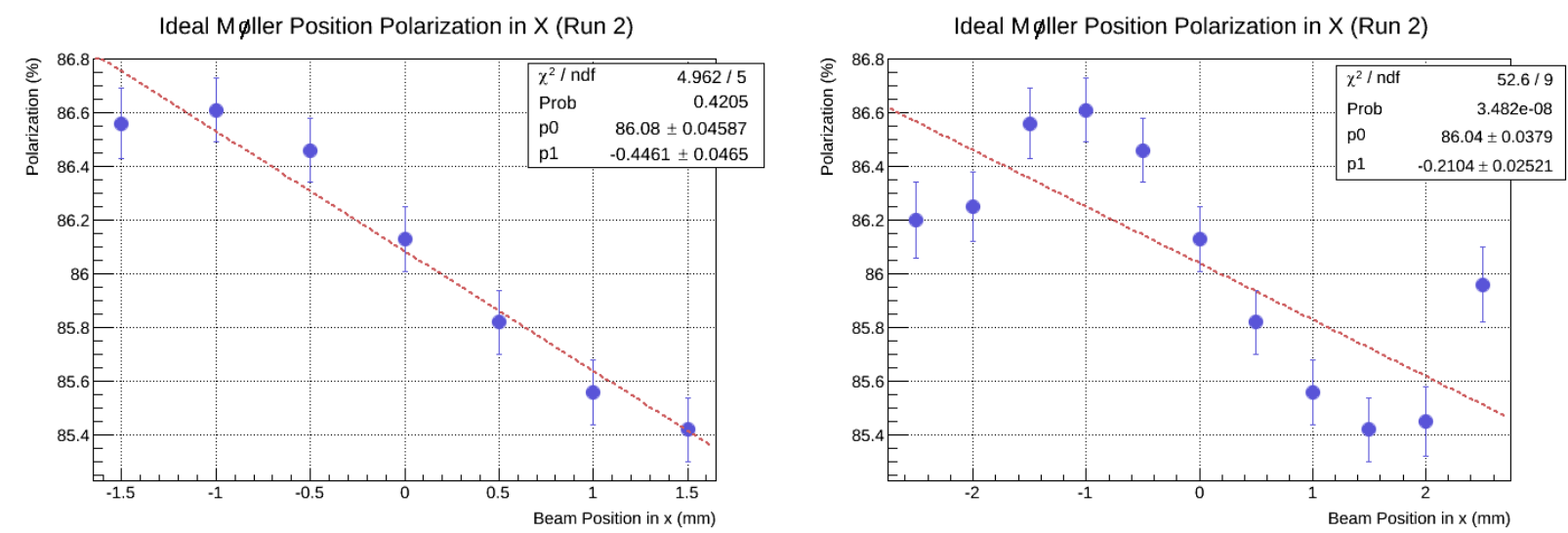

Figure 3: Simulation of measured dependence on target $x$-position. The fit from the left, including only points out to $\pm 1.5 \mathrm{~mm}$ was used. Right: If larger target values are used, non-linear behavior appears. Since only measurements at target positions $|x| \leq 1 \mathrm{~mm}$ are used, discrepancies at larger target distances are irrelevant.

$\mathrm{mm}$. To be conservative we doubled this estimate to $0.2 \mathrm{~mm}$ and then varied the $B P M$ positions individually to see the effect of calculated position difference at target.

To determine the overall uncertainty of target position and angle, the quadrature sum was taken between $\Delta P_{\text {calculation }}$ and $\Delta P_{\text {instrumental }}$.

\subsection{Quadrupole currents}

The quadrupole currents, and fields, also have some inherent uncertainty. Assuming the individual currents were uncorrelated leads to an over-conservative estimate. In reality, a slight mistune of one quad can be compensated by adjusting the field of the other.

It was assumed we knew the small quadrupole $(\mathrm{Q} 1)$ current to within $2 \%$ of itself, while we assumed we knew the large quad current (Q3) to within $1 \%$ of itself. For the study, each quadrupole current was individually shifted up-or-down by its respective uncertainty. Then, after observing the optical "tune" plot, the other quadrupole was changed to re-align to a nominal optical tune (see Figure 4).

The simulated study showed quantitative agreement with the data. In all, properly accounting for the correlation inherent in setting the quadrupole fields reduces the systematic uncertainty $(d A / A)$ from $0.45 \%$ to $0.08 \%$.

\subsection{Solenoid field scan}

One important systematic check is to study the measured polarization's dependence on the solenoid field. Since magnetic saturation in iron occurs at 2.2 Tesla, the measured polarization should be constant at all fields above 2.2 Tesla. Measurements are run at 3.5 T, ensuring magnetic saturation. Lastly, any foil tilt or warping will also become apparent, as saturation will not set in until higher magnetic fields are reached.

Figure 5 shows the calculated magnetization dependence for various foil angle tilts. Note that for slightly misaligned foils the measured value plateaus less quickly, and also to a lower value. Our 

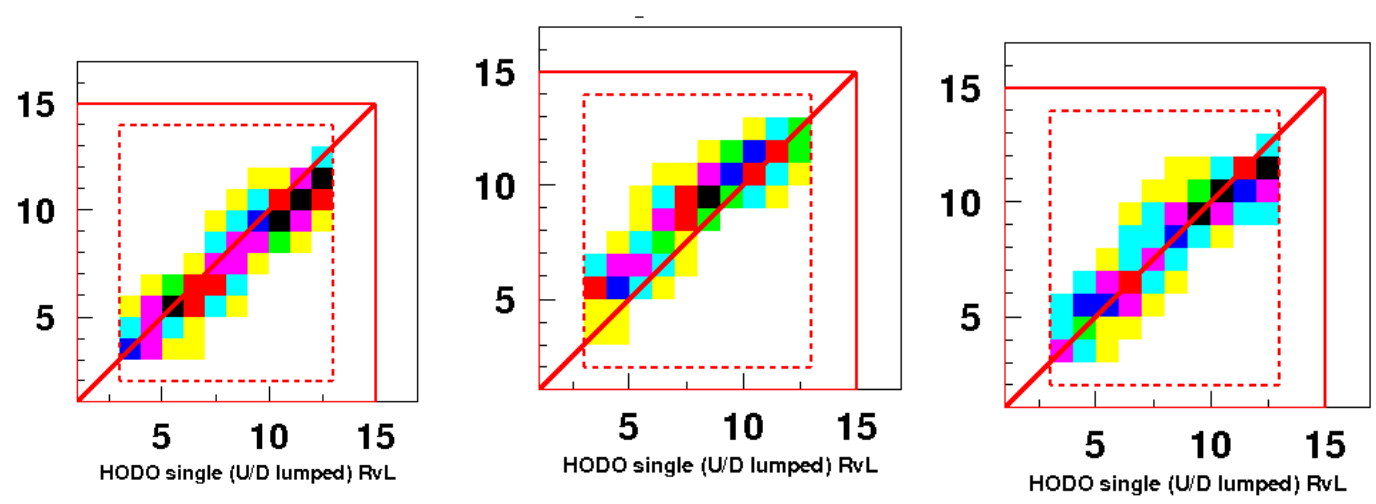

Figure 4: Tune plots taken from a December 2010 systematic study. Left: a nominal "tune" plot, showing a histogram of right versus left hodoscope arrays. Note the nice correlation between the two arrays. The red-line shows the ideal response. Center: A tune plot with the quad 3 field increased. Notice how the correlation "ridge" shifts upward. Right: After adjusting the smaller quad 1, the ridge shifts downward to its original position. Note that although the detector signal is still correctly centered on the red-line, the distribution appears to broaden slightly.
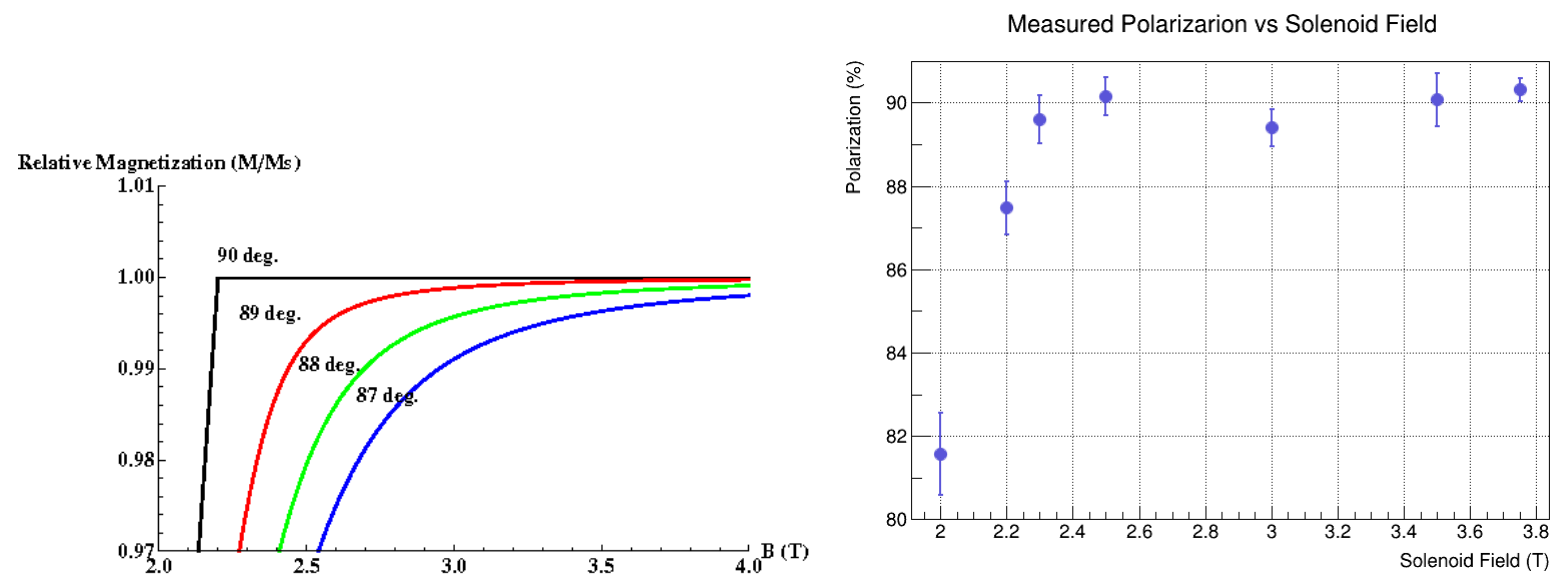

Figure 5: Left: calculated magnetization dependence on pure iron, shown for multiple foil tilts (in degrees). Note that for a perfectly perpendicular foil, the magnetization rises sharply at exactly 2.2T. Taken from [1]. Right: The actual study results performed during $Q_{\text {weak }}$. This behavior is consistent with a foil tilt of less than $2^{\circ}$.

measured polarization dependence is also shown and is consistent with less than a $1^{\circ}$ misalignment of the foil relative to the solenoid field. An upper limit of $2^{\circ}$ is conservatively assigned.

Lastly, the position of the solenoid itself is studied. As seen in Table 1, there is a $0.5 \mathrm{~mm}$ uncertainty in the absolute solenoid position. When the solenoid cools from room temperature, the actual position of the solenoid is only known at the $0.5 \mathrm{~mm}$ level. This slightly changes the axial field center, affecting the electron path slightly. A simulation-based study was done to determine the dependence on this degree of freedom. 


\subsection{Target Temperature Depolarization}

The Møller iron target magnetization decreases as the target foil heats up. Calculation of the Møller target heating has been written about in full before[1], and so will only be summarized here. Calculating the target temperature uncertainty is a two-step process: first the temperature rise of the target for different beam currents is calculated numerically using Mathematica; second, the actual demagnetization was determined using a fit to $M$ vs. $T$ data. The final uncertainty given in Table 1 is assumed $100 \%$ of the effect size.

\section{Summary}

The Hall C Møller polarimeter has successfully produced sub-percent electron polarization measurements during the $Q_{\text {weak }}$ experiment. Although previous experiments used this polarimeter, the systematic uncertainty requirements did not require the same level of precision; $Q_{\text {weak }}$ was the first Jefferson Lab experiment to successfully demonstrate sub-percent polarization measurements using a Møller device. Comprehensive studies of the polarimeter systematics were performed to meet this goal and several were discussed in Section 4. Most notably, an improved understanding of the correlation between magneto-optical elements (quadrupoles) resulted in a dramatic reduction in the $Q_{\text {weak }}$ uncertainty budget. The complete and final uncertainty budget for the Hall C Møller polarimeter is given in Table 1 .

\section{Acknowledgments}

The author would like to thank advisers David Gaskell of Jefferson Lab and David Armstrong of The College of William and Mary for their support and help preparing this report. This work was supported in part by the National Science Foundation grant NSF PHY-1068667. Jefferson Science Associates, LLC, operates Jefferson Lab for the U.S. DOE under U.S. DOE Contract No. DE-AC05-060R23177.

\section{References}

[1] M. Loppacher, Inaugral Thesis, Møller Polarimetry for CEBAF Hall C, University of Basel (1996).

[2] M. Hauger et al., Nucl. Instrum. Methods A 462, 382 (2001).

[3] D. Gaskell, Technical Report, Calculations of target heating for high current Møller polarimetry, Jefferson Lab, March 2004.

[4] D. Gaskell et al., Eur. Phys. J. A 32, 561 (2007).

[5] M. Poelker et al., Phys. Rev. ST Accel. Beams 10, 053502 (2007).

[6] M. Swartz et al., Nucl. Instrum. Methods A 363, 526 (1995).

[7] P. Royer, CERN-NUFACT-NOTE-11, CERN-OPEN-2000-324, CERN Technical Note (1999).

[8] K. L. Brown, Adv. Part. Phys. 1, 1 (1968);[Revised: SLAC-REPORT-R-075, Stanford University (1982)] 